\title{
Coulisses
}

Revue de théâtre

14 | Printemps 1996

Varia

\section{Tchekhov et son temps}

\section{Serge Rolet}

\section{OpenEdition}

Journals

Édition électronique

URL : http://journals.openedition.org/coulisses/4495

DOI : $10.4000 /$ coulisses.4495

ISSN : 2546-9460

\section{Éditeur}

Presses universitaires de Franche-Comté

\section{Édition imprimée}

Date de publication : 1 mai 1996

Pagination : 8-13

ISSN : 1150-594X

\section{Référence électronique}

Serge Rolet, « Tchekhov et son temps », Coulisses [En ligne], 14 | Printemps 1996, mis en ligne le 20 mars 2019, consulté le 22 octobre 2019. URL : http://journals.openedition.org/coulisses/4495 ; DOI : 10.4000/coulisses.4495

Ce document a été généré automatiquement le 22 octobre 2019

Coulisses 


\section{Tchekhov et son temps}

\section{Serge Rolet}

1 Dans les dernières années de sa vie, Tchekhov a été l'objet d'un respect, d'une admiration unanimes en Russie.

Cette reconnaissance allait même trop loin, au gré de certains de ses contemporains. Elle contenait une bonne dose d'hypocrisie. Ainsi, lors des funérailles de Tchekhov, un auteur tel que L. Andreïev avait été révolté par la tentative éhontée de récupération de la personne et de l'œuvre de Tchekhov par les représentants de l'esprit petit-bourgeois. Cette unanimité (entendons-nous, elle est quand même tardive : j'y reviendrai), cette reconnaissance universelle est curieuse. D'abord, l'œuvre de Tchekhov contient une critique quelquefois sévère de ses contemporains, et beaucoup, jusque dans les milieux intellectuels, pouvaient se sentir visés. Il y a là une question : comment pouvait-il dire des choses aussi sévères, et en même temps être apprécié de tous?

Ni Dostoïevski, ni Tolstoï, ni Gorki ne jouissent d'une pareille unanimité, d'une telle approbation.

Mais surtout, l'unanimité dont bénéficie Tchekhov est tardive : il y a deux périodes bien tranchées dans sa carrière, quant à la manière dont il était jugé par le public et la critique.

Jusqu'en 1890 à peu près, Tchekhov a été considéré comme inférieur à des écrivains comme Garchine et Nadson, auteurs aujourd'hui inconnus en dehors de la Russie. Il n'y a guère à l'époque que L. Tolstoï, pourtant si méfiant à l'égard de la jeune génération, si hostile à la Russie de ce temps, qui l'ait apprécié. "C'est à présent le règne du matérialisme, c'est-à-dire des femmes et des médecins ». Tchekhov était matérialiste et médecin ; pourtant Tolstoï reconnaissait la valeur de ses œuvres. Tchekhov est même le seul écrivain que Tolstoï ait considéré comme égal, voire supérieur à lui- même sur le plan artistique. 


\section{Tchekhov et la morosité des années 1880}

\section{Le « siècle de la dépression nerveuse »}

2 À la naissance de Tchekhov, en 1860, la Russie a un nouveau tsar, Alexandre II. L'événement qui donne le ton du règne, c'est l'abolition du servage en 1861. L'événement a une portée sociale et politique immense, et à sa suite, toute une série de réformes engagent la Russie - au moins le pense-t-on - sur la voie de la modernisation, du progrès. Selon Dostoïevski, par exemple, l'année 1861 ouvrait une «époque de passage vers des lendemains meilleurs».

À la mort de Dostoïevski, vingt ans plus tard, alors que Tchekhov commence à publier, l'époque des démocrates révolutionnaires, des populistes révolutionnaires est passée. Le " tsar libérateur ", Alexandre II, a été assassiné, son successeur s'engage sur la voie de la répression policière, sans que le peuple ni l'opinion ne réagissent: les Russes aspirent au calme, après l'agitation, la violence (attentats) des années 1870. La figure emblématique des années 1880, c'est Pobedonostsev, le ministre dont les «ailes de chouette " s'étendaient sur le pays. Politiquement, quand le jeune Tchekhov se lance dans la littérature, les temps sont à la dépression.

Sur le plan artistique, la dépression est également perceptible. Les grandes figures des années 40 et 60 ont quitté la scène: Tourgueniev, Dostoïevski, Ostrovski sont morts. Saltykov-Chtchedrine n'en a plus pour longtemps, de même que Gleb Ouspenski. Seul Tolstoï « faisait pour tout le monde », comme le dit Tchekhov avec quelque perfidie, car en réalité il n'écrivait presque plus de textes esthétiques, mais des articles et opuscules édifiants. Les années 1880-1890 sont perçues comme une époque de transition et de crise.

4 Dans le domaine des idées, dans le débat des intellectuels sur la société, c'est la même chose. Pour l'un des représentants des cercles cultivés et des "grosses revues » des deux capitales, Soloviev-Andreïevitch, par exemple, « l'époque a privé les gens de leur jeunesse. Le temps est à la boue sur les chemins, le temps est à l'automne ».

Dans un état d'esprit voisin, le philosophe Merejkovski publie en 1893 un essai qui a fait date, Des causes de la décadence et des nouveaux courants de la littérature contemporaine. Sur un ton différent de celui de Soloviev, Merejkovski joue dans le même registre : «Tout fond. Ce qui était blanc comme neige s'est transformé en une masse boueuse et friable » et ainsi de suite. Le siècle, pense-t-on alors, est à la nervosité.

Même Gorki, qui plaçait tant d'espoir dans l'avenir, dans l'homme nouveau, disait de ces années-là : «Je ne connais pas dans l'histoire russe de moment plus pénible que celui-ci ».

\section{L'état d'esprit de Tchekhov}

5 Tchekhov n'entre pas dans les débats sur «l'absence d'époque ", l'absence de perspectives: "À elle seule, la conviction que les années 1880 n'ont pas donné le moindre écrivain pourrait servir à remplir cinq volumes». Toute l'attitude de Tchekhov est là : le pessimisme de l'époque est un sujet d'observation sur le mode humoristique.

6 À Merejkovski, selon lequel le siècle est à la dépression nerveuse, Tchekhov répond (1891) : «Il n'y a pas de siècle de la nervosité » et précise : « Les gens vivent aujourd'hui 
comme ils vivaient avant, et les nerfs d'aujourd'hui ne sont pas moins solides que ceux d'Abraham, d'Isaac et de Jacob ». Quand Diaghilev lui propose de prendre aux côtés de Merejkovski la direction de la revue Le monde de l'art, il refuse : «Si nous poussons la brouette ensemble, ce ne sera pas dans la même direction ».

Tchekhov s'impatiente quand on évoque son "pessimisme »: "Moi, un pessimiste? Moi, je vois tout en noir?» Non qu'il trouve que tout va bien, mais «si la situation actuelle est mauvaise, celle d'hier était tout simplement horrible ». Tchekhov est issu d'une famille de moujiks: il sait ce que les réformes libérales d'Alexandre II ont apporté, il se rappelle les conditions de l'ancienne vie.

7 La conscience d'être dans un cul-de-sac, dans une époque privée de perspectives est limitée aux cercles littéraires, aux leaders des grands journaux. Dans le domaine scientifique, il n'y a pas de dépression. La fin du XIX ${ }^{e}$ siècle est riche de découvertes; certaines sont capitales (Lobatchevski, Mendeleev, Tsiolkovski).

Le grand historien de la culture Ovsianiko-Koulikovski estimait que les années 1880 étaient une période féconde de maturation intellectuelle, de redécouverte des grands classiques. Aujourd'hui, A. Soljénitsyne, grand connaisseur de la période, estime que les années 1880 sont les meilleures pour la pensée russe.

La position de Tchekhov quant au jugement porté sur la Russie de son temps n'a donc rien de commun avec le malaise des intellectuels. Tchekhov a plus d'affinités avec d'autres mondes, celui des scientifiques, par exemple.

La crise, donc, n'existe en fait que dans les cercles populistes : l'art littéraire, la vie intellectuelle, cherchaient des formes nouvelles, libérées des illusions du populisme, et allaient bientôt les trouver, avec Gorki, Tchekhov, et le symbolisme.

\section{Le courant littéraire auquel appartient Tchekhov}

8 Tchekhov est entré dans la carrière littéraire par la petite porte. Il ne faisait pas partie du milieu (celui de l'intelligentsia littéraire) dont on attendait de nouveaux écrivains. Longtemps, il n'a eu aucun lien avec ce milieu, et son entrée dans la "grande" littérature est exceptionnelle, quasi miraculeuse. Aucun de ses pareils n'a franchi la distance qui séparait la «petite littérature » de la grande.

Tchekhov a eu ses prédécesseurs et ses maîtres. À côté de la littérature concentrée uniquement sur les questions aiguës de la lutte sociale et politique, il y avait une autre littérature, qui se développait hors des cercles et des traditions intellectuelles. Elle était liée très fortement à la province, aux coins perdus de l'empire, à un monde que les écrivains de Pétersbourg et.de Moscou évitaient et qu'ils ignoraient presque complètement. Cette littérature n'enseignait ni ne professait rien de précis; elle se contentait de décrire la vie des Russes de l'époque, sans s'occuper des grandes questions. Vue de Pétersbourg et de Moscou, elle paraissait dépourvue d'idées, et «donc» privée de légitimité; on la méprisait. C'est à cette tradition-là, celle de Pissemski et de Leskov, que Tchekhov appartient. Il semble avoir pensé que cette littérature avait le droit d'exister, qu'il ne suffisait pas de décrire la Russie «en profondeur», comme Dostoïevski et Tolstoï avaient su le faire, mais aussi «en largeur ».

10 Résoudre les problèmes, c'était bien, mais il fallait tout de même commencer par recueillir le matériau le plus complet possible pour poser correctement les bonnes questions. La littérature noble à vocation sociale, philosophique, mettait la charrue 
avant les bœufs; en termes médicaux, elle rédigeait l'ordonnance avant de faire le diagnostic, et même, avant d'ausculter le patient (c'est-à-dire la Russie) pour savoir s'il était vraiment malade. Or la Russie, selon Tchekhov, n'est pas plus malade en 1880-1890 qu'à n'importe quelle autre période.

\section{Tchekhov et les grands débats de son temps}

\section{L'abstention de Tchekhov}

11 On a reproché à Tchekhov l'absence, ou l'insuffisance d'affirmation dans ses œuvres, le défaut de vision positive du monde, et surtout de propositions, de prescriptions. La reproduction des faits, semblait-t-il, obéissait chez lui au pur et simple hasard; Tchekhov n'expliquait rien. Tchekhov, c'est vrai, s'abstient de dire où est la vérité et plus encore, de dire où est le bien. Rien chez lui de messianique. Pour autant, ses œuvres ne sont pas dépourvues de message. Il y a dans ses récits et dans ses pièces une leçon quant à la manière de résister à l'oppression.

Malgré les sollicitations, Tchekhov refuse d'énoncer quelque programme que ce soit. « Il me semble que ce ne sont pas les écrivains qui doivent résoudre des questions telles que Dieu, le pessimisme, etc. L'affaire de l'écrivain est seulement de représenter les gens qui parlent de Dieu et du pessimisme ou qui y pensent, de quelles façons et dans quelles circonstances ils le font. L'artiste ne doit pas être le juge de ses personnages et de ce qu'ils disent, mais seulement le témoin impartial. J'ai entendu, entre deux Russes, une conversation sans suite et ne résolvant pas la question du pessimisme, et je dois reproduire cette conversation exactement comme je l'ai entendue. Les jurés, c'est-àdire les lecteurs, décideront. Mon rôle est seulement d'avoir du talent, c'est-à-dire de savoir distinguer les indices importants de ceux qui sont insignifiants, de savoir mettre en lumière des personnages, parler leur langue » (Lettre à Souvorine, 1888).

Selon Tchekhov, le lecteur peut être satisfait si la littérature se contente de poser correctement les problèmes; il n'exige pas de solution. "Il suffira qu'on désigne la maladie ; quant au traitement, Dieu sait lequel il faudrait appliquer ».

Cette position est en désaccord avec les gens de lettres de l'époque en Russie. Pour toutes sortes de raisons, les auteurs subissent une forte pression de la part de la critique et du public: on attend des écrivains qu'ils prennent parti dans les controverses politiques et sociales. Le débat d'idée impose à l'art littéraire son langage.

Sans remonter très loin dans l'histoire, on peut rappeler la vision de Tchernychevski. Selon Tchernychevski, «l'art, c'est la vie » : l'art ne doit pas simplement refléter la vie, mais il doit montrer le processus historique à l'œuvre dans la vie, il doit s'inscrire au sein de ce processus, être animé par la dynamique même de l'histoire. Chez Pissarev, disciple de Tchernychevski, on assiste à la " destruction de l'esthétique » : la spécificité de l'œuvre d'art est complètement niée. La "publitsistika », autrement dit l'idéologie dans le domaine intellectuel, le journalisme d'opinion, prime tout.

Dans les cercles littéraires soumis à l'influence du populisme tardif, on légifère sur l'attitude de l'auteur réaliste. Ainsi, le chef de file des populistes, Mikhaïlovski, estime que le processus social est comme un cortège, un mouvement de foule, et que l'écrivain peut choisir d'être "en tête" ou "en queue» de cortège : le contenu éthique et politique de son œuvre peut varier sensiblement d'un cas à l'autre. Mikhaïlovski dégage le concept de «réalité possible»: c'est elle que l'écrivain doit refléter. Korolenko 
préfère pour sa part l'avant-garde : pour lui l'écrivain doit montrer le chemin, il doit être la lumière qui brille dans le noir.

L'univers esthétique des années 1880 est ainsi fait que les auteurs de fiction sont sous l'empire des grandes revues. Les jeunes auteurs sont façonnés par les articles que leur consacrent les grands critiques. Les exigences de ces derniers ont quelque chose de tyrannique. Leur interprétation des œuvres est extrêmement réductrice, unilatérale, pauvre. On peut parler, bien avant l'époque soviétique, d'embrigadement de la littérature : la bataille littéraire se déroule camp contre camp.

Tchekhov oppose à cette entreprise de direction de conscience une résistance obstinée.

\section{La petite presse}

15 Le milieu intellectuel et littéraire, celui des journaux et des revues sérieux, de la critique engagée, celui où étaient débattus les grands problèmes de la société russe, Tchekhov le considère avec méfiance et sévérité. Il l'accuse d'oisiveté, de grossièreté, d'ignorance, de verbiage et d'enflure. Longtemps, il se contente de petits récits sans prétention, qu'il écrit avec facilité, rapidement, comme s'il n'avait pas l'intention de devenir un écrivain, avec tout le pathos qui va avec.

Ces petites pièces, parodies, anecdotes, humoresques, etc., paraissent dans un type particulier de publications, dans ce qu'on appelle la "petite presse ", par opposition aux grosses revues sérieuses ayant pignon sur rue. Ce point est capital, car la petite presse, justement, échappe, à cette époque, à la tyrannie des maîtres à penser.

L'apparition de la petite presse (comparable à ce qu'étaient au temps de Maupassant les journaux à un sou) remonte aux années 1870 . On regroupe sous la notion de petite presse des feuilles humoristiques destinées à la lecture en famille, la presse de « quai de gare » et les journaux de boulevard. Les titres sont nombreux, les tirages très élevés ; aux numéros réguliers (en règle générale hebdomadaires) de cette petite presse, il faut ajouter d'innombrables almanachs humoristiques paraissant dans les grandes villes. Certains de ces almanachs reprenaient les « humoresques » que Tchekhov donnait dans les feuilles hebdomadaires.

La petite presse n'a pas de relations avec les journaux et revues sérieux : les patrons, les auteurs, les lecteurs ne sont pas les mêmes. La petite presse est aux mains le plus souvent d'industriels, de maîtres de fabrique sans formation intellectuelle comparable à celle des dirigeants du Contemporain ou des Annales de la patrie. Les numéros, de grand format (in-4) font huit pages, ils comportent des rubriques régulières, un « courrier des lecteurs ", mais pas de débats idéologiques. Entre les titres, la concurrence est forte ; c'est la chasse aux abonnés, et la lutte pour les tirages est vive. On s'arrache les « bons " auteurs de récits humoristiques, d'anecdotes, de billets satiriques, de parodies, rémunérés cinq ou six kopeks la ligne. Tchekhov, lui, obtient huit kopeks de ses publications au journal Les Eclats. L'habitude dans la petite presse veut que les auteurs prennent des pseudonymes. Tchekhov s'en invente plus de cinquante (« le frère de mon frère "; "le médecin qui n'a pas de patient »; "celui qui s'est fait enlever la rate ", etc.). 


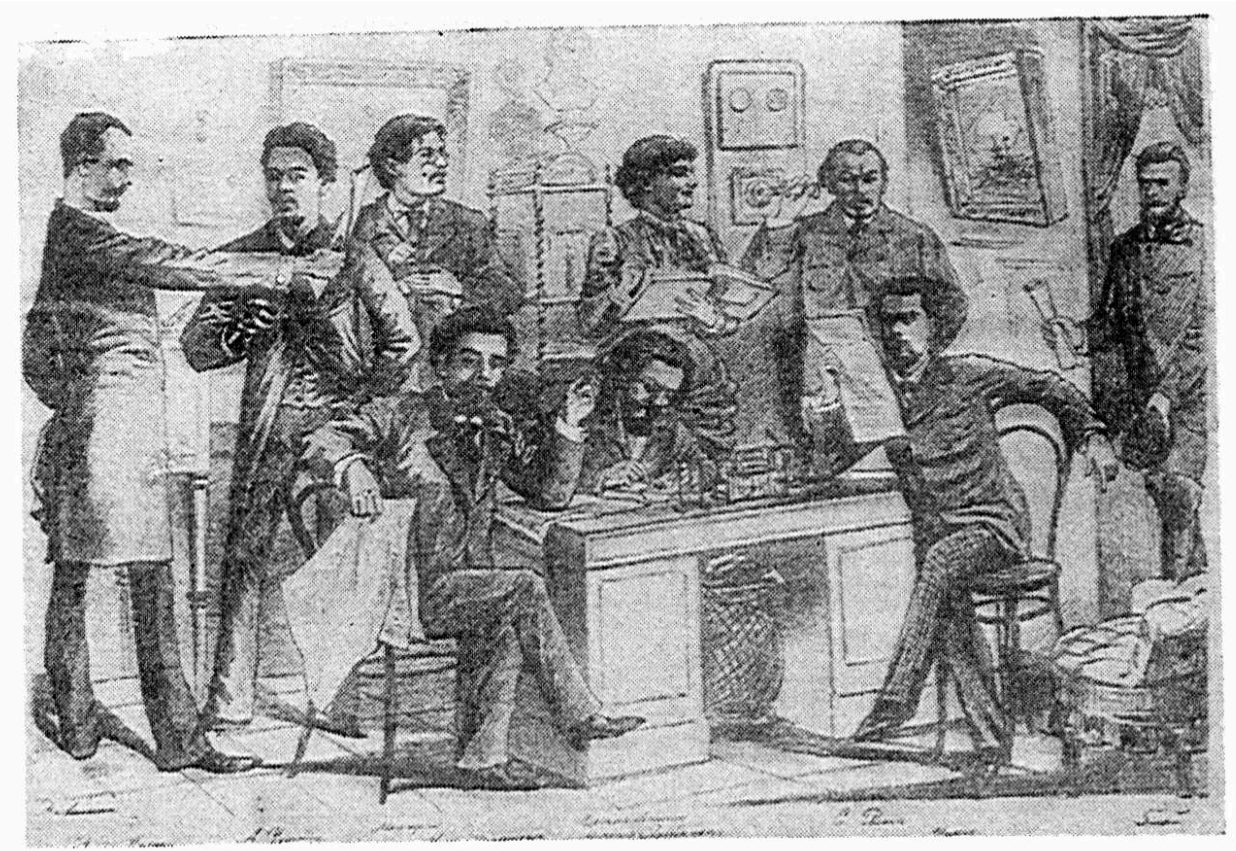

\section{Le rire comme résistance à l'oppression}

Ecrire pour la petite presse, traiter avec humour des faiblesses et des ridicules des Russes, cela n'empêche pas Tchekhov de s'intéresser aux grands problèmes. Sa correspondance, les souvenirs de ses contemporains, montrent qu'il n'a jamais perdu de vue ses origines. La misère, l'ignorance, les épidémies, les châtiments corporels font aussi partie de son univers. Tchekhov mesurait la force de la servitude et de la peur dans la mentalité des Russes. L'humour, la parodie, l'exagération sont pour lui autant d'armes contre les grands fléaux de la société.

« Nulle part l'autorité n'est aussi pesante que chez nous, les Russes, qui sommes écrasés par une servitude séculaire, et craignons la liberté ».

Il y a chez Tchekhov une psychologie de la peur, dont le personnage symbole est "l'homme dans un étui ", décrit comme un personnage "à tête de fouine ». La peur engendre la nullité, le ridicule.

Avec Tchekhov se clôt le thème de l'homme faible, du petit, apparu avec Gogol (Le manteau) et Dostoïevski (Les pauvres gens, Douce). On découvre, notamment dans ses récits, ce qui empêche l'homme d'être grand. Toute une série de nouvelles, de tendance satirique, rappelle Saltykov-Chtchedrine. Mais la plupart des récits de Tchekhov sont purement humoristiques. Sur ce point Tchekhov tranche sur ses contemporains : chez eux la nouvelle est un genre grave, sérieux, pénétré par les "grands problèmes ", par la conscience du devoir.

M. Gromov fait remonter le rire Tchekhovien à Hippocrate ; c'est peut-être excessif, mais on peut admettre l'idée que le rire Tchekhovien est lié à la formation de médecin de notre auteur. Tchekhov associe, c'est vrai, le rire à la santé ; il le "prescrit » à la manière d'une thérapeutique dans ses lettres à Olga Knipper, par exemple. On retrouve le rire, travesti, grimé, dans ce qui passe pour le « pessimisme » de Tchekhov. 
19 Le goût de Tchekhov pour l'humour se traduit dans son utilisation de la cacophonie, des lapsus, dans les erreurs de vocabulaire qu'il place dans la bouche de ses personnages d'origine étrangère (allemande le plus souvent). De tels traits apparaissent jusque dans les récits les plus graves, les plus émouvants, et dans les pièces. Ainsi, dans lonytch, l'intendant du domaine, voulant expliquer au propriétaire que les serrures sont abîmées et que les rideaux sont décrochés, a cette formule : "Les dénégations sont abîmées et la timidité s'est décrochée ». Les surnoms des personnages de Tchekhov contiennent souvent des jeux de mots, de même que leur discours : «Je suis un être humain, un être vivant, un substantif animé !» s'écrie l'un d'eux. Dans les nouvelles parues dans la petite presse, les jeux de mots, les plaisanteries, sont continuels : « Si ta femme t'a trahi, réjouis-toi que ce soit toi qu'elle ait trahi, et non pas la patrie » (pour «tromper » et " trahir », on emploie en russe le même verbe). Plusieurs des trouvailles de Tchekhov sont restées dans l'usage jusqu'à aujourd'hui.

En dehors de son caractère bénéfique pour la santé, le rire a aussi une valeur politique. Il est profondément démocratique : celui qui rit devient d'emblée l'égal de celui dont il rit. Rire, c'est se libérer de la peur, de l'oppression, de la pédagogie de Tolstoï et des populistes, une réaction contre le pharisaïsme de ceux qui, parlant d'un ton grave, ne font que perpétuer la peur, la servilité.

Il y a chez Tchekhov beaucoup de textes qui n'appartiennent pas à la "grande littérature », mais entre eux et la "grande littérature », il s'instaure un débat: les anecdotes, les «humoresques» de Tchekhov tournent en ridicule les hommes de l'époque et leurs petites difficultés, leurs incohérences, mais elles parodient aussi la " grande littérature ", avec ses héros complexes et tragiques.

\section{Le projet de Tchekhov : faire sortir la littérature du " tuyau de descente » où elle se tient confinée}

\section{Connaître la vie, voir le monde}

21 Tchekhov conseille au jeune Kouprine de voyager, de se montrer curieux. Il apprécie les premières œuvres de Gorki, qui évoque de manière neuve sa vie aventureuse.

Le regard de Tchekhov portait loin. Il admirait par exemple le grand explorateur Prjevalski. "J'aime infiniment les gens comme Prjevalski. (...) Leur personnalité apporte à la société la preuve vivante que, en dehors de ceux qui discutent de l'optimisme et du pessimisme, qui écrivent par ennui des mauvais récits, tirent des plans sur la comète, pondent des thèses à quatre sous, s'abandonnent à la débauche sous prétexte de refuser cette vie et mentent par intérêt, en dehors des sceptiques, des psychopathes, des jésuites, des philosophes, des libéraux et des conservateurs, il y a des hommes d'une autre trempe, des hommes capables d'exploits, capables de foi, qui suivent un but clair et conscient » (1898).

A côté des intellectuels russes de son temps, qui tiraient tout de leur esprit, et se montraient capables de dresser tout un tableau de la société sans rapport réel avec la réalité, ne faisant que projeter sur le réel une problématique propre à leur milieu, Tchekhov appelle au voyage, à la comparaison, à la découverte. Sakhalin, Ceylan, l'Europe occidentale le passionnent.

Le regard qu'il jette sur la Russie, sur le monde - en principe connu - est le même. J'ai fait allusion à la tradition provinciale dans laquelle s'inscrit Tchekhov. L'idée, de la part 
de Tchekhov était d'embrasser la réalité russe la plus large possible, de ne pas se limiter à tel ou tel milieu. Il y a quelque chose d'encyclopédique dans le tableau qu'il donne de la société. «J'ai dans la tête toute une armée d'hommes qui demandent à en sortir et attendent le signal », écrit-il en 1888. Dans le même esprit, il reproche à Korolenko de ne pas vouloir se séparer des détenus dont il dépeint l'existence épouvantable. Contre la fidélité à une cause, Tchekhov choisit la diversité. Dénoncer la barbarie de l'ordre policier, comme Korolenko, c'est bien ; s'y limiter, c'est moins bien.

\section{L'antitolstoïsme de Tchekhov}

Tchekhov a horreur de l'enfermement, du repli, des savoirs spécialisés. Rester enfermé dans un «tuyau de descente » (le mot est de lui), c'est se couper de la vie. Mais prôner le retour à la terre, comme le fait Tolstoï, c'est aussi, en un certain sens, se renfermer, craindre la vie.

Après avoir suivi quelque temps Toltstoï, Tchekhov se détache de lui ; il écrit en 1894 : «La morale de Tolstoï a fini de me toucher; au fond je n'ai pour elle nulle complaisance... C'est du sang de moujik que j'ai dans les veines. Aussi, rien qui m'étonne dans les vertus du moujik. Pour moi Toltstoï est déjà loin, il n'est plus dans mon âme, il s'en est allé en disant : “je laisse votre maison vide (...)". »

Tchekhov rejette la posture tolstoïenne : la figure d'ermite barbu en chemise, poussant la charrue et affrontant seul les puissances déchaînées du Mal. A ses yeux l'héroïsme de Tolstoï a tout de la mise en scène. Tolstoï était un grand noble; le respect dont il jouissait le mettait à l'abri des attaques qui auraient balayé à coup sûr tout autre que lui. Son attitude ne pouvait être un exemple.

Tolstoï et Tchekhov à Gaspra (Crimée)

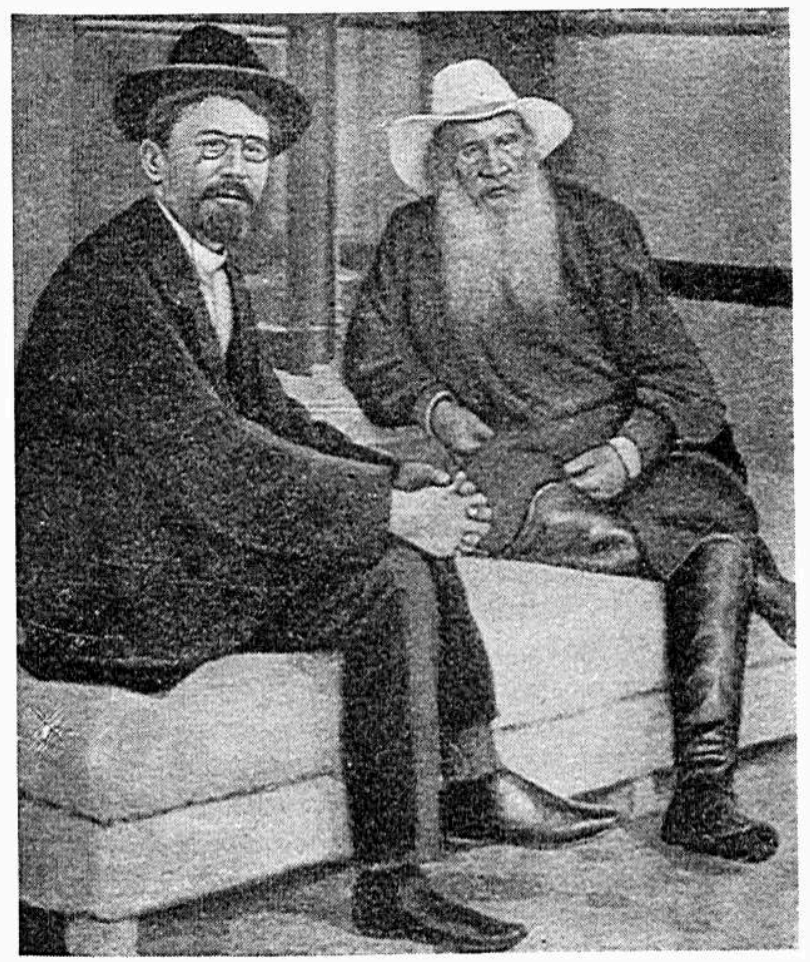



de l'individuel et du social, de l'historique et de l'intime, du général et du particulier. Elles sont thématisées dans l'œuvre de L. Tolstoï. Poser correctement les problèmes, pour Tchekhov, c'est, là encore, dépasser les oppositions du tolstoïsme. Dans Guerre et paix, «la vraie vie» (la vie personnelle) s'oppose au cours de l'histoire. Pour bon nombre d'auteurs de son temps, le souci de l'universel conduit à oublier le particulier : la littérature n'est plus qu'idées générales, allégories. Cette opposition du général et du particulier, Tchekhov la gomme : il refuse absolument de choisir l'un des termes contre l'autre. Pour lui, le moindre incident de la vie personnelle du premier venu résonne des grands problèmes historiques et sociaux. Il faut considérer la Russie globalement.

En ceci réside la spécificité de Tchekhov : dans le fait que le diagnostic le plus aigu, le plus juste, le plus convaincant, soit exprimé par les signes les plus ténus, les plus insignifiants. Les comportements les plus ordinaires, les moindres gestes, les intonations, la démarche des personnages sont essentiels. L'incapacité de tel obscur personnage à échapper à la vulgarité en dit plus long sur la peur de la liberté et la servitude séculaire que la représentation naturaliste des conditions de vie dans les campagnes ou dans les faubourgs.

La facilité de Tchekhov, l'abondance de ses écrits, sont liées au fait qu'il était le premier à s'intéresser au monde des petits faits sans importance, aux concours de circonstances, aux lapsus, aux maladresses, à tout ce qui dans le quotidien est risible ou bizarre. Tchekhov décide que ce qui n'a aucune importance est digne de la plus grande attention.

À ce jeu, la littérature fait ventre de tout. Le moindre incident fournit de quoi faire un récit : un petit fonctionnaire qui éternue sur le crâne luisant d'un général, cela donne le sujet d'une nouvelle. Un jour qu'il parlait avec Korolenko de sa conception de la nouvelle, Tchekhov, à titre de preuve par l'exemple, proposa à Korolenko d'écrire un récit sur le cendrier qu'ils avaient sous les yeux, là, sur la table. Et le lendemain, il lui tendit le manuscrit : le récit était prêt. 